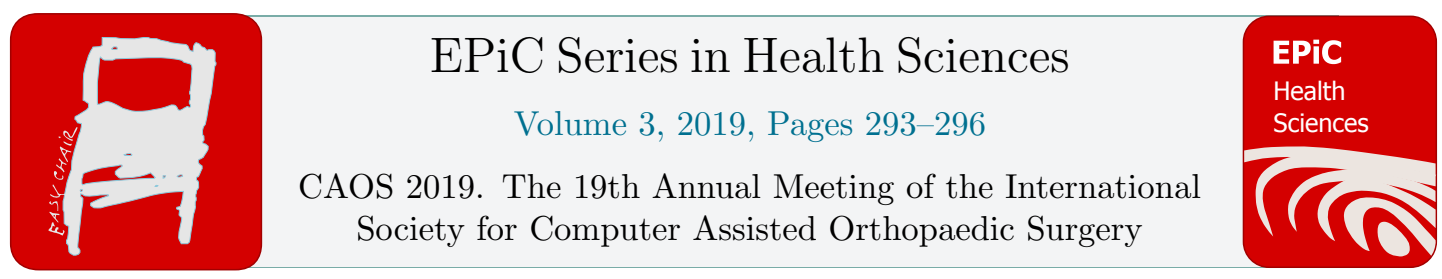

\title{
Development and validation of image-based subject-specific skeletal models of spinal deformity patients for use in motion analysis
}

\author{
Thomas Overbergh ${ }^{1,3 *}$, Pieter Severijns ${ }^{1}$, Lieven Moke ${ }^{1,2}$, Ilse Jonkers ${ }^{4}$, \\ Lennart Scheys $1,2,3$ \\ ${ }^{1}$ Institute for Orthopaedic Research and Training (IORT), ${ }^{2}$ University Hospitals Leuven, \\ ${ }^{3}$ Department of Regeneration and Development, K.U. Leuven, ${ }^{4}$ Department of Biomedical \\ Kinesiology, K.U. Leuven, Belgium; \\ Corresponding author: Thomas.Overbergh@kuleuven.be
}

\begin{abstract}
Conventional radiographic analysis in spinal deformity only quantifies the static skeletal body structure and no conclusions can be drawn with respect to functional abilities. The aim of this work was to introduce subject-specific, skeletal full spine multibody models in a spinal deformity population for quantifying their full dynamic function. To accomplish this, the creation of a subject-specific model as well as its use with optical motion capture was validated.

As such, this work puts within reach the extension of the current state-of-the-art clinical management from static based concepts with concepts that integrate dynamic functional data.
\end{abstract}

\section{Introduction}

Static and two dimensional (2D) imaging-based measurements form the basis of current quantitative diagnosis, pre-operative planning and clinical management of patients with spinal deformities (Terran, 2013). However, since back pain and spine-related complications arise most frequently during dynamic activities of daily living (ADL), these approaches inherently fail to capture the complete functionality of the patient (Moke, 2018). Musculoskeletal modeling and multi-body simulation have clear potential to improve the insight into the impact of the deformity and its treatment by quantifying biomechanical parameters, such as joint kinematics and kinetics, during gait and other ADL. However, a workflow for multi-body analyses (MBA) in spinal deformity has not been standardized or validated yet. MBA are typically driven by optical motion capture data, tracking markers positioned on the skin above an anatomical landmark. However, the absence of sufficient palpable vertebral anatomical landmarks often results in incorrect and too few marker placements, thereby leading to erroneous marker-based scaling, 
static initialization and dynamic tracking of the spine using a generic skeletal model of the healthy spine (Overbergh, 2016).

Therefore, the first objective of this study was to develop and validate custom software for reconstruction of a subject-specific skeletal model of the spine based on biplanar radiographic imaging, which replaces the erroneous marker-based scaling of generic models. The second objective was to in vivo quantify the vertebral tracking accuracy of the thus generated model and associated custom marker protocol in spinal deformity patients.

\section{Methods}

\subsection{Development and validation of an image-based modeling platform}

A user-friendly application, based on biplanar images (EOS System, Paris) taken at low radiation and in an upright, load-bearing position, was developed to introduce subject-specificity to any generic OpenSim (Delp, 2007) model. Three dimensional (3D) reconstructions were obtained using the direct linear transform principle (Abdel-Aziz, 2015) on both spatially calibrated images. Iteratively matching the 2D projections of CT-segmented bone geometry (of which the radiographic fan-beam effects were taken into account) on both radiograph planes allowed subject-specific realignment of the bony geometry (Fig.1). Furthermore, the software allowed radiograph-based definition of virtual markers relative to the underlying bones, thereby personalizing also the marker placement.

In order to quantify the accuracy of this personalization system, it was first applied on a plastinated cadaver thus ensuring the spinal alignment in the supine position was identical to the upright, loadbearing position. The individual vertebrae of the cadaver were obtained using computed tomography (CT), and segmented thereafter using Mimics 19.0 (Materialise, Belgium). The spinal alignment was then reconstructed onto the upright biplanar EOS images. The relative transformation matrix at each vertebral level was used to calculate the position and orientation error between the original CT-based alignment and the reconstructed EOS-based alignment.

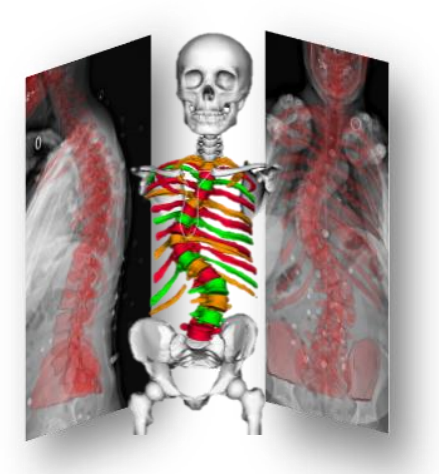

Figure 1: Spatially calibrated EOS images allow 3D reconstruction of the spinal alignment in a loadbearing position.

\subsection{Quantification of marker-based vertebral tracking capacity}

To determine the capability of spine markers to track the vertebrae, retroreflective markers were attached to nine subjects with varying degrees of spinal deformity by an experienced physiotherapist, according to the marker protocol shown in Fig.2A. In order to obtain 3D information about the spinal alignment, biplanar EOS images were obtained for all subjects in an upright load-bearing position (Fig.2B), and a bended position (Fig.2C).

Separate skeletal models of the spine were created based on the 'upright' and the 'bended' positions (Fig.2D). The intervertebral pin joints were centered and aligned with their adjacent endplate geometries. Markers were extracted from the bended model and used as inputs for simulations performed on the upright model, using the inverse kinematics tool in OpenSim. The 3D location and 
orientation of the individual vertebrae of this 'simulated' bended model were compared to the 'personalized' bended model, considered the ground truth.
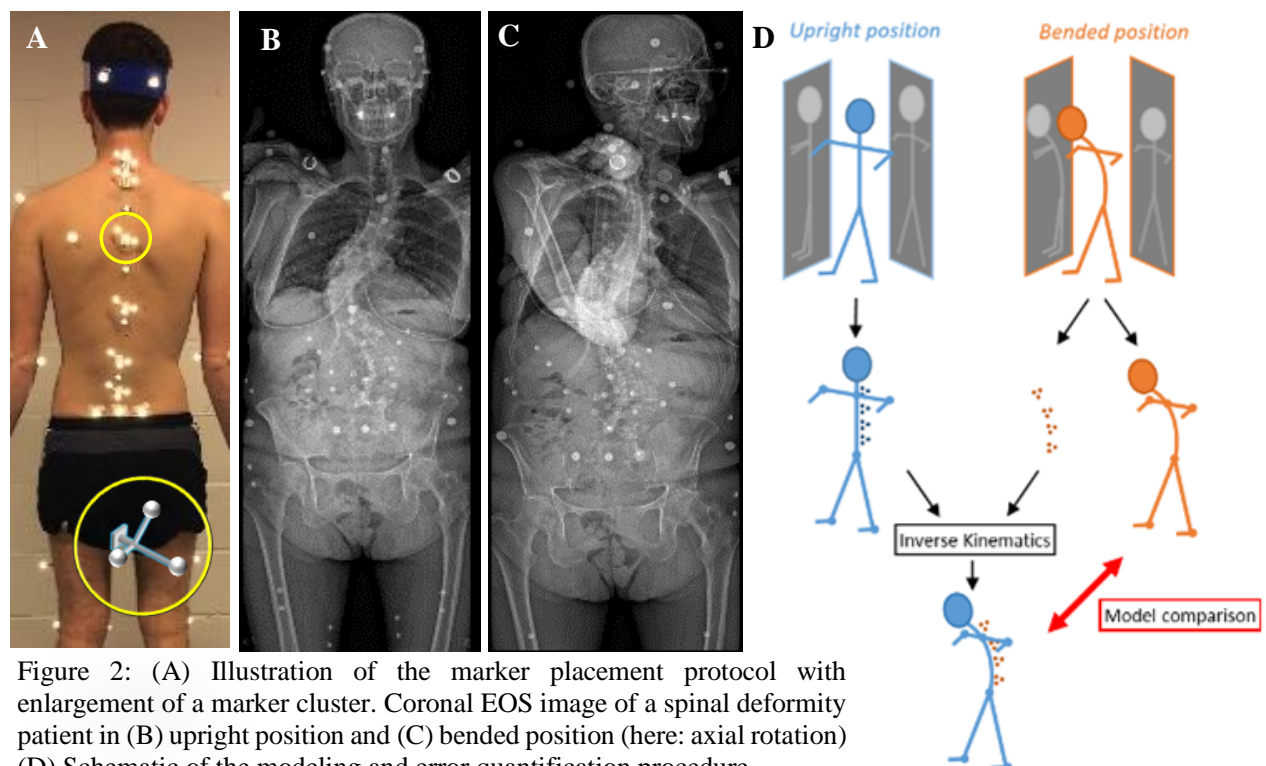

Figure 2: (A) Illustration of the marker placement protocol with
enlargement of a marker cluster. Coronal EOS image of a spinal deformity patient in (B) upright position and (C) bended position (here: axial rotation) (D) Schematic of the modeling and error quantification procedure.

\section{Results}

The average position error (SD) introduced during personalization of the plastinated cadaver for the anteroposterior, mediolateral and inferosuperior directions was $0.83 \mathrm{~mm}(0.67), 1.59 \mathrm{~mm}(0.83)$ and $0.71 \mathrm{~mm}(0.59)$, respectively. The corresponding average orientation errors were $1.25^{\circ}(1.25), 1.63^{\circ}$ (1.44) and $2.27^{\circ}(1.63)$, respectively.

The average vertebral tracking accuracy in the anteroposterior, mediolateral and inferosuperior directions was $3.22 \mathrm{~mm}(2.54), 4.76 \mathrm{~mm}(4.19)$ and $2.78 \mathrm{~mm}$ (3.11), respectively. The error for the orientations is for a rotation in: the frontal plane $3,56^{\circ}(3.31)$, the transverse plane $5,61^{\circ}(4.27)$ and the sagittal plane $2,98^{\circ}(2.29)$.

\section{Discussion}

Low errors in location and orientation of the vertebrae confirmed the performance of the custom software in personalizing the spinal alignment of generic models based on biplanar imaging, thereby facilitating MBA of spinal deformities. As the plastinated cadaver was devoid of internal organs that normally occlude the spinal region, thereby providing much clearer images than those obtained in vivo, these errors may be larger in real patients. However, in vivo, CT reconstruction is precluded as ground truth as the associated supine position will introduce differences in spinal alignment with the weightbearing position to be reconstructed.

The custom marker design and placement protocol successfully tackled the issue of marker-based actuation of the model. The reported errors could be explained as a combination of skin motion artifacts on the markers, the use of generic kinematic interpolation values (Overbergh, 2018) in a pathological 
Development and validation of image-based subject-specific skeletal models of ... T. Overbergh et al.

population, and smaller errors in the creation of the ground truth model using the software. Further statistical tests were not conducted owing to the small test population, considering the executed motion, and variability in subject BMI and spinal deformity, which are expected to influence skin motion artefact, and consequentially, the vertebral tracking capacity.

With the custom software, model and marker protocol, the kinematics during every recorded motion can be analyzed within the documented ranges of accuracy. This work will also allow the extension of the current static 2D radiographic evaluation with in silico determined dynamic parameters based on joint kinematics and kinetics, which is suggested by Moke (2018) to potentially contribute to clinical decision-making and management.

\section{References}

Abdel-Aziz, Y. I., \& Karara, H. M. (2015). Direct Linear Transformation from Comparator Coordinates into Object Space Coordinates in Close-Range Photogrammetry. Photogrammetric Engineering \& Remote Sensing, 81(2), 103-107.

Delp, S. L., Anderson, F. C., Arnold, A. S., Loan, P., Habib, A., John, C. T., ... Thelen, D. G. (2007). OpenSim: open-source software to create and analyze dynamic simulations of movement. IEEE Transactions on Bio-Medical Engineering, 54(11), 1940-1950.

Moke, L. (2018). Determinants of health-related quality of life in patients with adult spinal deformity: Additional value of dynamic biomechanical measures. KU Leuven Thesis.

Overbergh, T., Pieter, S., Mariska, W., Moke, L., Vanpeteghem, A., Jonkers, I., \& Scheys, L. (2018). Quantification of kinematic error in multi-body simulations of spinal deformity subjects. ORS 2018 Annual Meeting Poster No . 1185, 7(1185), 2018.

Overbergh, T., Wesseling, M., Severijns, P., Moke, L., Jonkers, I., \& Scheys, L. (2016). Personalized musculoskeletal modeling of spinal deformities based on stereoradiographic images: use for biomechanical analysis of posture and motion. Belgium: 15th National Day on Biomedical Engineering.

Terran, J., Schwab, F., Shaffrey, C. I., Smith, J. S., Devos, P., Ames, C. P., ... Lafage, V. (2013). The SRS-schwab adult spinal deformity classification: Assessment and clinical correlations based on a prospective operative and nonoperative cohort. Neurosurgery, 73(4), 559-568. 